\title{
Treatment of corneal rust rings with desferrioxamine
}

\author{
P. J. NORTH
}

The Royal Berkshire Hospital, Reading

Desferrioxamine (Desferal, GIBA) eyedrops have been used for a number of ocular lesions caused by iron-containing compounds (Galin, Harris, and Papariello, 1965; Wise, I966; Valvo, 1967; McGuinness and Knight-Jones, 1968). This paper describes the results of treating 36 patients with rust staining of the cornea and a complication possibly due to this drug which has not previously been described.

\section{Clinical observations}

In this series, treatment was confined to corneal rust rings (after surgical removal of the original foreign body if necessary). The patient was prescribed gutt. desferrioxamine four times a day and reviewed regularly. The results were as follows:

\begin{tabular}{lr} 
Rust removed & 27 \\
Rust remaining, no symptoms & 4 \\
$\begin{array}{l}\text { Rust remaining, with symptoms } \\
\quad \text { (after } 7 \text { days treatment) }\end{array}$ & 2 \\
Other & 3 \\
\hline Total & 36
\end{tabular}

The two patients with remaining rust and symptoms had the rust surgically removed and became symptom-free.

In the last group, one patient complained of continued irritation in the eye for which no cause could be found. The other two patients developed corneal ulceration in the area of the original foreign body; although at this time there was no remaining rust, there was a heavy flare and cells in the aqueous. Both were treated with gutt. atropine I per cent., an antibiotic ointment, and padding. The ulceration healed in both cases within 3 days.

\section{Discussion}

No cases of reactions to desferrioxamine given locally have previously been reported. Two patients in this series developed corneal ulceration, which settled quickly, leaving no visual disability. There were no cases of allergy. Further observations are required to determine whether there is a true causative relationship. 
McGuinness and Knight-Jones (1968) have reported that there is no significant difference in efficacy between surgical and medical removal. However, desferrioxamine presents two advantages:

(x) With the wider geographical spacing of ophthalmic units in the periphery, many non-ophthalmologists would be competent to remove the original foreign body and treat remaining rust with desferrioxamine, thus saving many patients a long journey.

(2) If the drops can be instilled into the eye of a young child, the use of general anaesthesia may be avoided.

My thanks are due to Mr. G. T. W. Cashell, Mr. E. W. Dorrell, and Mr. J. McMillan for permission to report cases under their care, and to Dr. D. M. Burley of CIBA Laboratories for supplies of gutt. desferrioxamine.

\section{References}

Galin, M. A., harris, L. s., and Papariello, G. J. (1965) Arch. Ophthal. (Chicago), 74, 674 MCGUINNESS, R., and KNIGHT-JONES, D. (1968) Brit. F. Ophthal., 52, 777

valvo, A. (1967) Amer. F. Ophthal., 63, 98

Wise, J. B. (1966) Arch. Ophthal. (Chicago), 75, 698 